\title{
Attracting Periodic Cycles for an Optimal Fourth-Order Nonlinear Solver
}

\author{
Mi Young Lee and Changbum Chun \\ Department of Mathematics, Sungkyunkwan University, Suwon 440-746, Republic of Korea \\ Correspondence should be addressed to Changbum Chun, cbchun@skku.edu
}

Received 28 November 2011; Accepted 14 February 2012

Academic Editor: Khalida Inayat Noor

Copyright (C) 2012 M. Y. Lee and C. Chun. This is an open access article distributed under the Creative Commons Attribution License, which permits unrestricted use, distribution, and reproduction in any medium, provided the original work is properly cited.

We consider an optimal fourth-order method for solving nonlinear equations and construct polynomials such that the rational map arising from the method applied to these polynomials has an attracting periodic orbit of any prescribed period.

\section{Introduction}

Recently, an optimal fourth-order iterative method to find a simple root $\rho$, that is, $f(\rho)=0$ and $f^{\prime}(\rho) \neq 0$, of a nonlinear equation $f(x)=0$, which is given by

$$
x_{n+1}=x_{n}-\frac{16 f\left(x_{n}\right) f^{\prime}\left(x_{n}\right)}{-5 f^{\prime 2}\left(x_{n}\right)+30 f^{\prime}\left(x_{n}\right) f^{\prime}\left(y_{n}\right)-9 f^{\prime 2}\left(y_{n}\right)},
$$

where $y_{n}=x_{n}-(2 / 3)\left(f\left(x_{n}\right) / f^{\prime}\left(x_{n}\right)\right)$ was proposed in [1] and its dynamics behavior was investigated and analyzed in detail. By an optimal method, we mean a multipoint method without memory which requires $n+1$ functional evaluations per iteration, but achieves the order of convergence $2^{n}$ [2].

The method (1.1) may be used to approximate both real and complex roots of the nonlinear equation with $x_{0} \in \mathbb{C}$. If the initial guess $x_{0}$ is chosen sufficiently near a zero of $f$, then an iterative method is expected to converge. This is, however, not true in general if there exists an attracting periodic orbit or cycle of period $k \geq 2$ (whose definition will be introduced below). If the initial guess happens to be chosen from the basin of attraction of an attractive periodic cycle, the sequence $\left\{x_{n}\right\}_{n=0}^{\infty}$ converges to the attractive cycle, not to a zero of $f$ since any root of $f(x)=0$ may be considered as a period orbit of period 1 . Thus the existence of attracting periodic cycles of period greater than or equal to 2 could interfere with an iterative method searching for a root of the nonlinear equation. As a result, 
it has been an important concern from practical aspect in iteration theory to construct specific polynomials for a given method such that the map arising from the iterative method applied to the polynomials has an attractive periodic orbit. In this direction there was some result in [3] where attractive periodic orbits of any prescribed period were constructed for some classical third-order methods. Motivated by this, in this paper we extend the construction of attractive periodic cycles of any prescribed period to higher-order iterative methods. To this end, we will recall some preliminaries, see for example Milnor [4], Amat et al. [5], and Chun et al. [1]. Let $R: \widehat{\mathbb{C}} \rightarrow \widehat{\mathbb{C}}$ be a rational map on the Riemann sphere.

Definition 1.1. For $z \in \widehat{\mathbb{C}}$ we define its orbit as the set

$$
\operatorname{orb}(z)=\left\{z, R(z), R^{2}(z), \ldots, R^{n}(z), \ldots\right\}
$$

Definition 1.2. A point $z_{0}$ is a fixed point of $R$ if $R\left(z_{0}\right)=z_{0}$.

Definition 1.3. A periodic point $z_{0}$ of period $m$ is such that $R^{m}\left(z_{0}\right)=z_{0}$ where $m$ is the smallest such integer. The set of the $m$ distinct points $\left\{z, R(z), R^{2}(z), \ldots, R^{m-1}(z)\right\}$ is called a periodic cycle.

Remark 1.4. If $z_{0}$ is periodic of period $m$ then it is a fixed point for $R^{m}$.

Definition 1.5. If $z_{0}$ is a periodic point of period $m$, then the derivative $\left(R^{m}\right)^{\prime}\left(z_{0}\right)$ is called the eigenvalue of the periodic point $z_{0}$.

Remark 1.6. By the chain rule, if $z_{0}$ is a periodic point of period $m$, then its eigenvalue is the product of the derivatives of $R$ at each point on the orbit of $z_{0}$, and we have

$$
\left(R^{m}\right)^{\prime}\left(z_{0}\right)=\left(R^{m}\right)^{\prime}\left(z_{1}\right)=\cdots=\left(R^{m}\right)^{\prime}\left(z_{n-1}\right),
$$

that is, all the points of a cycle have the same eigenvalue.

We classify the fixed points of a map based on the magnitude of the derivative.

Definition 1.7. A point $z_{0}$ is called attracting if $\left|R^{\prime}\left(z_{0}\right)\right|<1$, repelling if $\left|R^{\prime}\left(z_{0}\right)\right|>1$, and neutral if $\left|R^{\prime}\left(z_{0}\right)\right|=1$. If the derivative is zero then the point is called superattracting.

Definition 1.8. The Julia set of a nonlinear map $R(z)$, denoted $J(R)$, is the closure of the set of its repelling periodic points. The complement of $J(R)$ is the Fatou set $\mathbb{F}(R)$.

By its definition, $J(R)$ is a closed subset of $\widehat{\mathbb{C}}$. A point $z_{0}$ belongs to the Julia set if and only if dynamics in a neighborhood of $z_{0}$ displays sensitive dependence on the initial conditions, so that nearby initial conditions lead to wildly different behavior after a number of iterations. As a simple example, consider the map $R(z)=z^{2}$ on $\widehat{\mathbb{C}}$. The entire open disk is contained in $\mathbb{F}(R)$, since successive iterates on any compact subset converge uniformly to zero. Similarly the exterior is contained in $\mathbb{F}(R)$. On the other hand if $z_{0}$ is on the unit circle than in any neighborhood of $z_{0}$ any limit of the iterates would necessarily have a jump discontinuity as we cross the unit circle. Therefore $J(R)$ is the unit circle. Such smooth Julia sets are exceptional. 
Lemma 1.9 (Invariance lemma (Milnor [4])). The Julia set $J(R)$ of a holomorphic map $R: \widehat{\mathbb{C}} \rightarrow \widehat{\mathbb{C}}$ is fully invariant under $R$. That is, $z$ belongs to $J$ if and only if $R(z)$ belongs to $J$.

Lemma 1.10 (Iteration Lemma). For any $k>0$, the Julia set $J\left(R^{k}\right)$ of the $k$-fold iterate coincides with $J(R)$.

Definition 1.11. If $O$ is an attracting periodic orbit of period $m$, we define the basin of attraction to be the open set $A \in \widehat{\mathbb{C}}$ consisting of all points $z \in \widehat{\mathbb{C}}$ for which the successive iterates $R^{m}(z), R^{2 m}(z), \ldots$ converge towards some point of $O$.

The basin of attraction of a periodic orbit may have infinitely many components.

Definition 1.12. The immediate basin of attraction of a periodic orbit is the connected component containing the periodic orbit.

Lemma 1.13. Every attracting periodic orbit is contained in the Fatou set of $R$. In fact the entire basin of attraction A for an attracting periodic orbit is contained in the Fatou set. However, every repelling periodic orbit is contained in the Julia set.

\section{Attractive Cycles Results}

Let us consider the map $R_{f}$ associated to $f$

$$
R_{f}(z)=z+\frac{16 f(z) f^{\prime}(z)}{5 f^{\prime 2}(z)-30 f^{\prime}(z) f^{\prime}(y)+9 f^{\prime 2}(y)}
$$

where

$$
y=z-\frac{2}{3} \frac{f(z)}{f^{\prime}(z)}
$$

for which the optimal method (1.1) is written as

$$
x_{n+1}=R_{f}\left(x_{n}\right), \quad n \geq 0 .
$$

Toward the aim to construct periodic orbits of any prescribed period for the method (1.1), we have the following characterization.

Proposition 2.1. Let $\Omega=\left\{x_{1}, x_{2}, \ldots, x_{n}\right\}$ be a set of $n$ distinct complex numbers, and let $f$ be a complex analytic function. Then $\Omega$ is periodic orbit of period $n$ of iteration function $R_{f}$ if and only if

$$
\begin{gathered}
\frac{16 f^{\prime}\left(x_{i}\right) f\left(x_{i}\right)}{x_{i+1}-x_{i}}=5 f^{\prime 2}\left(x_{i}\right)-30 f^{\prime}\left(x_{i}\right) f^{\prime}\left(y_{i}\right)+9 f^{\prime 2}\left(y_{i}\right), \quad i=1,2, \ldots, n-1, \\
\frac{16 f^{\prime}\left(x_{n}\right) f\left(x_{n}\right)}{x_{1}-x_{n}}=5 f^{\prime 2}\left(x_{n}\right)-30 f^{\prime}\left(x_{n}\right) f^{\prime}\left(y_{n}\right)+9 f^{\prime 2}\left(y_{n}\right),
\end{gathered}
$$

where $y_{i}=x_{i}-(2 / 3)\left(f\left(x_{i}\right) / f^{\prime}\left(x_{i}\right)\right), i=1,2, \ldots, n$. 
Proof. Assume that $\Omega$ is a periodic orbit of $R_{f}$. Then $R_{f}\left(x_{i}\right)=x_{i+1}$ for $i=1,2, \ldots, n-1$ and $R_{f}\left(x_{n}\right)=x_{1}$. We have

$$
\begin{gathered}
x_{i+1}=x_{i}+\frac{16 f^{\prime}\left(x_{i}\right) f\left(x_{i}\right)}{5 f^{\prime 2}\left(x_{i}\right)-30 f^{\prime}\left(x_{i}\right) f^{\prime}\left(y_{i}\right)+9 f^{\prime 2}\left(y_{i}\right)}, \quad i=1,2, \ldots, n-1, \\
x_{1}=x_{n}+\frac{16 f^{\prime}\left(x_{n}\right) f\left(x_{n}\right)}{5 f^{\prime 2}\left(x_{n}\right)-30 f^{\prime}\left(x_{n}\right) f^{\prime}\left(y_{n}\right)+9 f^{\prime 2}\left(y_{n}\right)} .
\end{gathered}
$$

Therefore, we obtain

$$
\begin{gathered}
x_{i+1}-x_{i}=\frac{16 f^{\prime}\left(x_{i}\right) f\left(x_{i}\right)}{5 f^{\prime 2}\left(x_{i}\right)-30 f^{\prime}\left(x_{i}\right) f^{\prime}\left(y_{i}\right)+9 f^{\prime 2}\left(y_{i}\right)}, \quad i=1,2, \ldots, n-1, \\
x_{1}-x_{n}=\frac{16 f^{\prime}\left(x_{n}\right) f\left(x_{n}\right)}{5 f^{\prime 2}\left(x_{n}\right)-30 f^{\prime}\left(x_{n}\right) f^{\prime}\left(y_{n}\right)+9 f^{\prime 2}\left(y_{n}\right)} .
\end{gathered}
$$

Conversely, suppose that $f$ satisfies condition (2.4). Then we easily have

$$
\begin{gathered}
R_{f}\left(x_{i}\right)=x_{i}+\frac{16 f^{\prime}\left(x_{i}\right) f\left(x_{i}\right)}{\left(16 f^{\prime}\left(x_{i}\right) f\left(x_{i}\right)\right) /\left(x_{i+1}-x_{i}\right)}=x_{i+1}, \quad i=1,2, \ldots, n-1, \\
R_{f}\left(x_{n}\right)=x_{n}+\frac{16 f^{\prime}\left(x_{n}\right) f\left(x_{n}\right)}{\left(16 f^{\prime}\left(x_{n}\right) f\left(x_{n}\right)\right) /\left(x_{1}-x_{n}\right)}=x_{1} .
\end{gathered}
$$

Proposition 2.2. For any positive integer $n \geq 2$, there exists a polynomial $f(x)$ of degree less than or equal to $3 n-1$ for which $R_{f}$ has a periodic orbit of period $n$.

Proof. Let $x_{1}, x_{2}, \ldots, x_{n}$ be a given a set of $n$ distinct complex numbers, let $z_{1}, z_{2}, \ldots, z_{n}$ be a set of $n$ nonzero complex numbers, and let $s_{1}, s_{2}, \ldots, s_{n}$ be another set of nonzero complex numbers such that

$$
\begin{gathered}
y_{i} \neq y_{j} \quad \forall i \neq j, \\
y_{i} \neq x_{j} \quad \forall i, j=1,2, \ldots, n, \\
15^{2}-9\left(5 s_{i}^{2}-\frac{16 s_{i} z_{i}}{x_{i+1}-x_{i}}\right) \geq 0, \quad i=1,2, \ldots, n-1, \\
15^{2}-9\left(5 s_{n}^{2}-\frac{16 s_{n} z_{n}}{x_{1}-x_{n}}\right) \geq 0
\end{gathered}
$$

where $y_{i}=x_{i}-(2 / 3)\left(z_{i} s_{i}\right)$. 
Assume that there exists a polynomial $f(x)$ such that

$$
\begin{gathered}
f\left(x_{i}\right)=z_{i}, \quad i=1,2, \ldots, n, \\
f^{\prime}\left(x_{i}\right)=s_{i}, \quad i=1,2, \ldots, n, \\
9 f^{\prime 2}\left(y_{i}\right)-30 s_{i} f^{\prime}\left(y_{i}\right)+5 s_{i}^{2}=\frac{16 s_{i} z_{i}}{x_{i+1}-x_{i}}, \quad i=1,2, \ldots, n-1, \\
9 f^{\prime 2}\left(y_{n}\right)-30 s_{n} f^{\prime}\left(y_{n}\right)+5 s_{n}^{2}=\frac{16 s_{n} z_{n}}{x_{1}-x_{n}} .
\end{gathered}
$$

Then by Proposition 2.1, $\Omega=\left\{x_{1}, x_{2}, \ldots, x_{n}\right\}$ is a periodic orbit of period $n$ of $R_{f}$. Using (2.9) and condition (2.8) yields

$$
\begin{gathered}
f^{\prime}\left(y_{i}\right)=\frac{15 \pm \sqrt{15^{2}-9\left(5 s_{i}^{2}-\left(16 s_{i} z_{i} /\left(x_{i+1}-x_{i}\right)\right)\right)}}{9} \quad i=1,2, \ldots, n-1, \\
f^{\prime}\left(y_{n}\right)=\frac{15 \pm \sqrt{15^{2}-9\left(5 s_{n}^{2}-\left(16 s_{n} z_{n} /\left(x_{1}-x_{n}\right)\right)\right)}}{9} .
\end{gathered}
$$

We now show that such a polynomial exists. For this, we use the Hermite interpolation procedure. We begin the construction by writing $f(x)$ as

$$
f(x)=\sum_{i=1}^{3 n} a_{i} f_{i}(x)
$$

where the functions $f_{i}(x)$ are polynomials defined as follows:

$$
\begin{aligned}
f_{1}(x) & =1 \\
f_{2}(x) & =\left(x-x_{1}\right) \\
f_{3}(x) & =\left(x-x_{1}\right)^{2} \\
f_{4}(x) & =\left(x-x_{1}\right)^{2}\left(x-y_{1}\right)^{2}, \\
f_{5}(x)= & \left(x-x_{1}\right)^{2}\left(x-y_{1}\right)^{2}\left(x-x_{2}\right), \\
f_{6}(x)= & \left(x-x_{1}\right)^{2}\left(x-y_{1}\right)^{2}\left(x-x_{2}\right)^{2}, \\
f_{7}(x)= & \left(x-x_{1}\right)^{2}\left(x-y_{1}\right)^{2}\left(x-x_{2}\right)^{2}\left(x-y_{2}\right)^{2}, \\
& \vdots \\
f_{3 n}(x)= & \left(x-x_{1}\right)^{2}\left(x-y_{1}\right)^{2}\left(x-x_{2}\right)^{2}\left(x-y_{2}\right)^{2} \cdots\left(x-x_{n}\right)^{2} .
\end{aligned}
$$


To determine the polynomial $f(x)$ we must find suitable coefficients $a_{i}$ for $i=1$, $2, \ldots, 3 n$. For this we must solve a linear system of $3 n$ equations with $3 n$ unknown of the form $A X=B$. The matrix $A$ associated with the linear system of equations is given by

$$
A=\left(\begin{array}{ccccc}
f_{1}\left(x_{1}\right) & f_{2}\left(x_{1}\right) & f_{3}\left(x_{1}\right) & \cdots & f_{3 n}\left(x_{1}\right) \\
f_{1}^{\prime}\left(x_{1}\right) & f_{2}^{\prime}\left(x_{1}\right) & f_{3}^{\prime}\left(x_{1}\right) & \cdots & f_{3 n}^{\prime}\left(x_{1}\right) \\
f_{1}^{\prime}\left(y_{1}\right) & f_{2}^{\prime}\left(y_{1}\right) & f_{3}^{\prime}\left(y_{1}\right) & \cdots & f_{3 n}^{\prime}\left(y_{1}\right) \\
f_{1}\left(x_{2}\right) & f_{2}\left(x_{2}\right) & f_{3}\left(x_{2}\right) & \cdots & f_{3 n}\left(x_{2}\right) \\
f_{1}^{\prime}\left(x_{2}\right) & f_{2}^{\prime}\left(x_{2}\right) & f_{3}^{\prime}\left(x_{2}\right) & \cdots & f_{3 n}^{\prime}\left(x_{2}\right) \\
f_{1}^{\prime}\left(y_{2}\right) & f_{2}^{\prime}\left(y_{2}\right) & f_{3}^{\prime}\left(y_{2}\right) & \cdots & f_{3 n}^{\prime}\left(y_{2}\right) \\
\cdots & \cdots & \cdots & \cdots & \cdots \\
f_{1}^{\prime}\left(y_{n}\right) & f_{2}^{\prime}\left(y_{n}\right) & f_{3}^{\prime}\left(y_{n}\right) & \cdots & f_{3 n}^{\prime}\left(y_{n}\right)
\end{array}\right)
$$

and the column vectors $X, B \in \mathbb{R}^{n}$ are given by $X=\left(a_{1}, a_{2}, \ldots, a_{3 n}\right)^{T}$ and $B=$ $\left(f\left(x_{1}\right), f^{\prime}\left(x_{1}\right), f^{\prime}\left(y_{1}\right), \ldots, f\left(x_{n}\right), f^{\prime}\left(x_{n}\right), f^{\prime}\left(y_{n}\right)\right)^{T}$. The components of the upper triangle of the matrix $A$ are zero, and the components of the diagonal of $A$ are nonzero. Therefore, the linear system $A X=B$ has a unique solution.

Example 2.3. Let us construct a polynomial $f(x)$ for which the iterative method $R_{f}$ has a periodic orbit of period 2. For this, we let $x_{1}=0, x_{2}=1, z_{1}=f\left(x_{1}\right)=2, z_{2}=f\left(x_{2}\right)=$ $2, s_{1}=f^{\prime}\left(x_{1}\right)=2$ and $s_{2}=f^{\prime}\left(x_{2}\right)=2$. Then we have $y_{1}=x_{1}-(2 / 3)\left(z_{1} / s_{1}\right)=-2 / 3, y_{2}=$ $x_{2}-(2 / 3)\left(z_{2} / s_{2}\right)=1 / 3, f^{\prime}\left(y_{1}\right)=\left(15 \pm \sqrt{15^{2}-9\left(5 s_{1}^{2}-16 s_{1} z_{1} /\left(x_{2}-x_{1}\right)\right)}\right) / 9=22 / 3,-2 / 3$, and $f^{\prime}\left(y_{2}\right)=\left(15 \pm \sqrt{15^{2}-9\left(5 s_{2}^{2}-16 s_{2} z_{2} /\left(x_{1}-x_{2}\right)\right)}\right) / 9=14 / 3,2$. We construct a polynomial $f(x)=\sum_{i=1}^{6} a_{i} f_{i}(x)$, where $f_{1}(x)=1, f_{2}(x)=x, f_{3}(x)=x^{2}, f_{4}(x)=x^{2}(x+(2 / 3))^{2}, f_{5}(x)=$ $x^{2}(x+(2 / 3))^{2}(x-1), f_{6}(x)=x^{2}(x+(2 / 3))^{2}(x-1)^{2}$. Then matrix $A$ is given by

$$
A=\left(\begin{array}{cccccc}
f_{1}\left(x_{1}\right) & 0 & 0 & 0 & 0 & 0 \\
f_{1}^{\prime}\left(x_{1}\right) & f_{2}^{\prime}\left(x_{1}\right) & 0 & 0 & 0 & 0 \\
f_{1}^{\prime}\left(y_{1}\right) & f_{2}^{\prime}\left(y_{1}\right) & f_{3}^{\prime}\left(y_{1}\right) & 0 & 0 & 0 \\
f_{1}\left(x_{2}\right) & f_{2}\left(x_{2}\right) & f_{3}\left(x_{2}\right) & f_{4}\left(x_{2}\right) & 0 & 0 \\
f_{1}^{\prime}\left(x_{2}\right) & f_{2}^{\prime}\left(x_{2}\right) & f_{3}^{\prime}\left(x_{2}\right) & f_{4}^{\prime}\left(x_{2}\right) & f_{5}^{\prime}\left(x_{2}\right) & 0 \\
f_{1}^{\prime}\left(y_{2}\right) & f_{2}^{\prime}\left(y_{2}\right) & f_{3}^{\prime}\left(y_{2}\right) & f_{4}^{\prime}\left(y_{2}\right) & f_{5}^{\prime}\left(y_{2}\right) & f_{6}^{\prime}\left(y_{2}\right)
\end{array}\right) .
$$


By evaluating $A$ we obtain

$$
A=\left(\begin{array}{cccccc}
1 & 0 & 0 & 0 & 0 & 0 \\
0 & 1 & 0 & 0 & 0 & 0 \\
0 & 1 & -\frac{4}{3} & 0 & 0 & 0 \\
1 & 1 & 1 & \frac{25}{9} & 0 & 0 \\
0 & 1 & 2 & \frac{80}{9} & \frac{25}{9} & 0 \\
0 & 1 & \frac{2}{3} & \frac{8}{9} & -\frac{13}{27} & \frac{20}{81}
\end{array}\right) .
$$

Now we consider the system of linear equations $A X=B$, where $X=\left(a_{1}, a_{2}, a_{3}\right.$, $\left.a_{4}, a_{5}, a_{6}\right)^{T}$ and $B=\left(f\left(x_{1}\right), f^{\prime}\left(x_{1}\right), f^{\prime}\left(y_{1}\right), f\left(x_{2}\right), f^{\prime}\left(x_{2}\right), f^{\prime}\left(y_{2}\right)\right)^{T}$. Here, we can take $B=$ $(2,2,22 / 3,2,2,14 / 3)^{T}$. Hence we have the following system:

$$
\left(\begin{array}{cccccc}
1 & 0 & 0 & 0 & 0 & 0 \\
0 & 1 & 0 & 0 & 0 & 0 \\
0 & 1 & \frac{-4}{3} & 0 & 0 & 0 \\
1 & 1 & 1 & \frac{25}{9} & 0 & 0 \\
0 & 1 & 2 & \frac{80}{9} & \frac{25}{9} & 0 \\
0 & 1 & \frac{2}{3} & \frac{8}{9} & \frac{-13}{27} & \frac{20}{81}
\end{array}\right)\left(\begin{array}{c}
a_{1} \\
a_{2} \\
a_{3} \\
a_{4} \\
a_{5} \\
a_{6}
\end{array}\right)=\left(\begin{array}{c}
2 \\
2 \\
\frac{22}{3} \\
2 \\
2 \\
\frac{14}{3}
\end{array}\right)
$$

whose solution is $X=(2,2,-4,18 / 25,72 / 125,12582 / 625)^{T}$. Therefore, the polynomial $f$ is given by $f(x)=2+2 x-4 x^{2}+(18 / 25) x^{2}(x+(2 / 3))^{2}+(72 / 125) x^{2}(x+2 / 3)^{2}(x-1)+$ $(12582 / 625) x^{2}(x+2 / 3)^{2}(x-1)^{2}=2+2 x+(3132 / 625) x^{2}-(14808 / 625) x^{4}+(5872 / 625) x^{3}-$ $(8028 / 625) x^{5}+(12582 / 625) x^{6}$. From $(2.1)$, we obtain $R_{f}(0)=1$ and $R_{f}(1)=0$. So, $\Omega=\{0,1\}$ is a periodic orbit of period 2 for the method $R_{f}(x)$.

Proposition 2.4. Let $f(x)$ be a polynomial for which $R_{f}$ has a periodic orbit of period $n$, say $\Omega=$ $\left\{x_{1}, x_{2}, \ldots, x_{n}\right\}$. If $f^{\prime \prime}\left(x_{i}\right)=0=f^{\prime \prime}\left(y_{i}\right)$ and $f^{\prime}\left(x_{i}\right)=f^{\prime}\left(y_{i}\right)$ or $f^{\prime \prime}\left(x_{i}\right)=0=f^{\prime \prime}\left(y_{i}\right)$ and $7 f^{\prime}\left(x_{i}\right)=$ $3 f^{\prime}\left(y_{i}\right)$ for some $i=1,2, \ldots, n$, then $\Omega$ is an superattracting periodic orbit of period $n$ for $R_{f}$.

Proof. Without loss of generality, we may assume that $f^{\prime \prime}\left(x_{1}\right)=0=f^{\prime \prime}\left(y_{1}\right)$ and $f^{\prime}\left(x_{1}\right)=f^{\prime}\left(y_{1}\right)$. By the chain rule, we have

$$
\left(R_{f}^{n}\right)^{\prime}\left(x_{1}\right)=R_{f}^{\prime}\left(x_{n}\right) R_{f}^{\prime}\left(x_{n-1}\right) R_{f}^{\prime}\left(x_{n-2}\right) \cdots R_{f^{\prime}}\left(x_{1}\right) .
$$


Note that on differentiating (2.1) we have

$$
\begin{aligned}
R_{f^{\prime}}(x)=[ & \left.f^{\prime}(x)\left(5 f^{\prime 2}(x)-30 f^{\prime}(x) f^{\prime}(y)+9 f^{\prime 2}(y)\right)^{2}\right]^{-1} \\
& \times\left[f^{\prime}(x)\left(5 f^{\prime 2}(x)-30 f^{\prime}(x) f^{\prime}(y)+9 f^{\prime 2}(y)\right)\left(21 f^{\prime}(x)^{2}-30 f^{\prime}(x) f^{\prime}(y)\right)\right. \\
& +9 f^{\prime 2}(y)+16 f(x) f^{\prime \prime}(x) \\
& \quad-16 f(x)\left[10 f^{\prime 3}(x) f^{\prime \prime}(x)-30 f^{\prime 2}(x) f^{\prime \prime}(x) f^{\prime}(y)\right. \\
& \left.\left.-2\left(f^{\prime 2}(x)+2 f(x) f^{\prime \prime}(x)\right)\left(5 f^{\prime}(x) f^{\prime \prime}(y)-3 f^{\prime}(y) f^{\prime \prime}(y)\right)\right]\right] .
\end{aligned}
$$

We therefore obtain $R_{f}^{\prime}\left(x_{1}\right)=0$. By $(2.17)$ we conclude that $\left(R_{f}^{n}\right)^{\prime}\left(x_{1}\right)=0$.

\section{Acknowledgments}

This research was supported by Basic Science Research Program through the National Research Foundation of Korea (NRF) funded by the Ministry of Education, Science and Technology (2011-0025877).

\section{References}

[1] C. Chun, M. Y. Lee, B. Neta, and J. Džunić, "On optimal fourth-order iterative methods free from second derivative and their dynamics," Applied Mathematics and Computation, vol. 218, no. 11, pp. 6427$6438,2012$.

[2] H. T. Kung and J. F. Traub, "Optimal order of one-point and multipoint iteration," Journal of the Association for Computing Machinery, vol. 21, pp. 643-651, 1974.

[3] S. Amat, S. Busquier, and S. Plaza, "A construction of attracting periodic orbits for some classical thirdorder iterative methods," Journal of Computational and Applied Mathematics, vol. 189, no. 1-2, pp. 22-33, 2006.

[4] J. Milnor, Dynamics in One Complex Variable, vol. 160 of Annals of Mathematics Studies, Princeton University Press, Princeton, NJ, USA, 3rd edition, 2006.

[5] S. Amat, S. Busquier, and S. Plaza, "Iterative root-finding methods," Unpublished Report, 2004. 


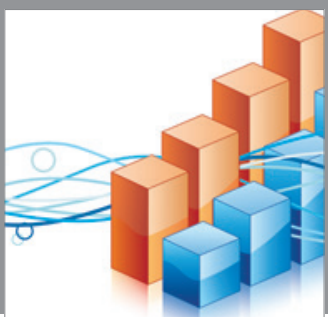

Advances in

Operations Research

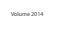

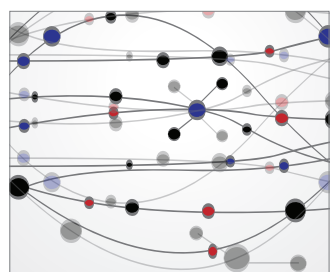

\section{The Scientific} World Journal
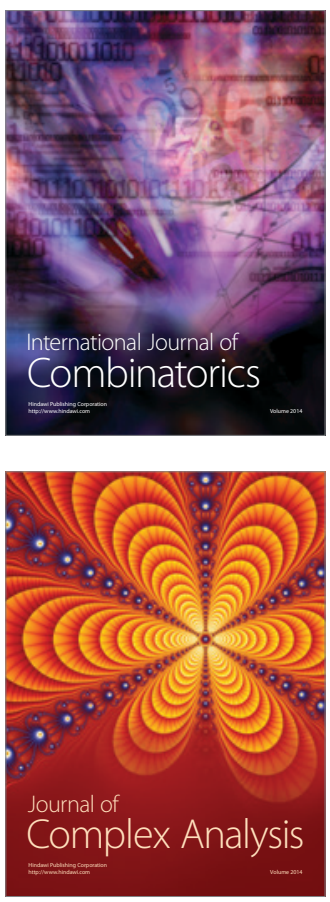

International Journal of

Mathematics and

Mathematical

Sciences
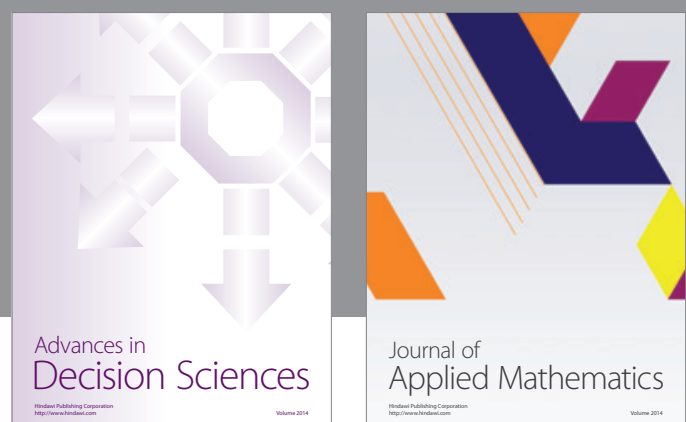

Journal of

Applied Mathematics
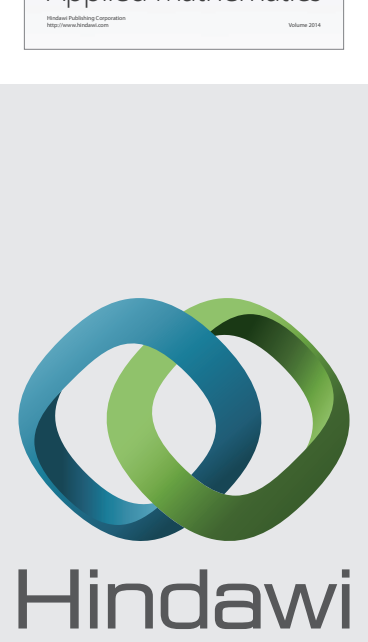

Submit your manuscripts at http://www.hindawi.com
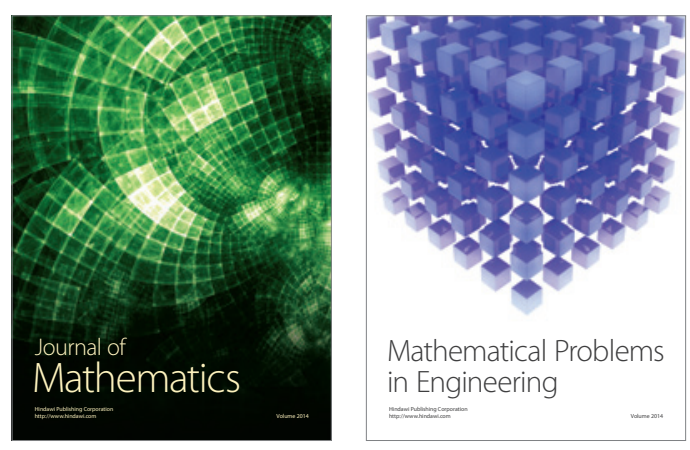

Mathematical Problems in Engineering
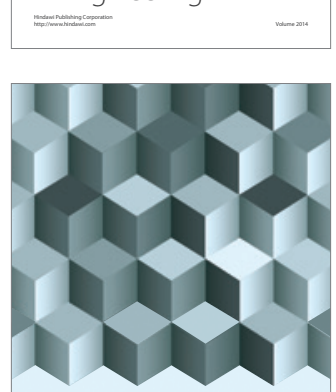

Journal of

Function Spaces
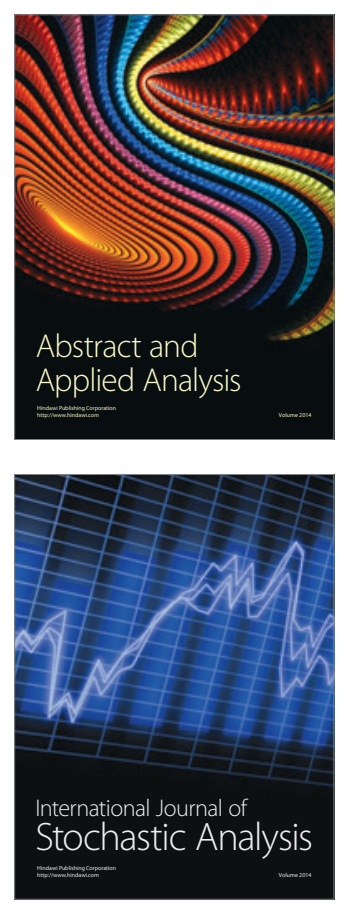

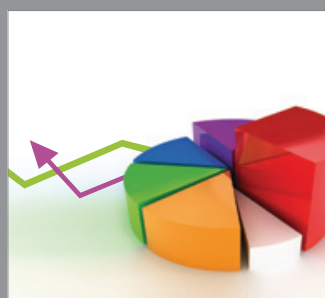

ournal of

Probability and Statistics

Promensencen
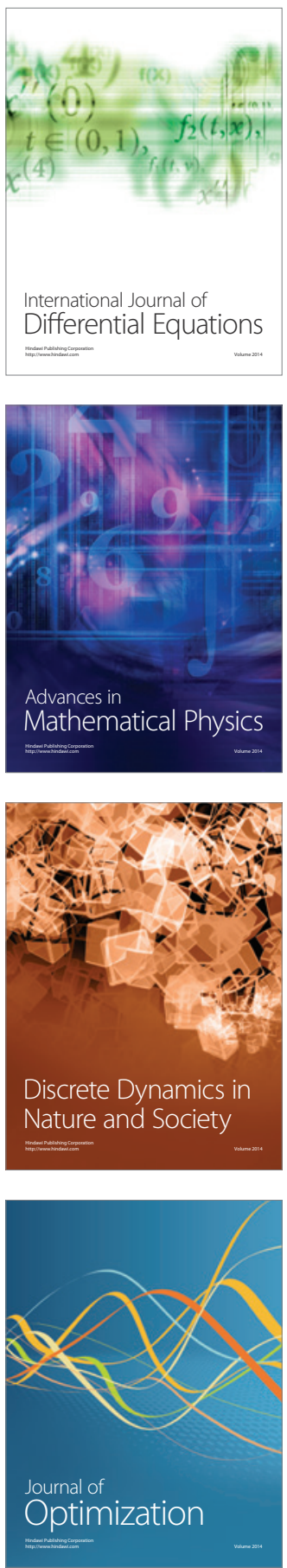